Relations industrielles

Industrial Relations

\title{
Le syndicalisme québécois, par Louis-Marie Tremblay, Montréal, Presses de l'Université de Montréal, 1972, 288 pp.
}

\section{Bernard Solasse}

Volume 28, numéro 1, 1973

URI : https://id.erudit.org/iderudit/028373ar

DOI : https://doi.org/10.7202/028373ar

Aller au sommaire du numéro

Éditeur(s)

Département des relations industrielles de l'Université Laval

ISSN

0034-379X (imprimé)

1703-8138 (numérique)

Découvrir la revue

Citer ce compte rendu

Solasse, B. (1973). Compte rendu de [Le syndicalisme québécois, par Louis-Marie Tremblay, Montréal, Presses de l’Université de Montréal, 1972, 288 pp.]

Relations industrielles / Industrial Relations, 28(1), 207-208.

https://doi.org/10.7202/028373ar

Tous droits réservés (C) Département des relations industrielles de l'Université Laval, 1973
Ce document est protégé par la loi sur le droit d'auteur. L’utilisation des services d'Érudit (y compris la reproduction) est assujettie à sa politique d'utilisation que vous pouvez consulter en ligne.

https://apropos.erudit.org/fr/usagers/politique-dutilisation/ 


\section{RECENSIONS}

Le syndicalisme québécois, par LouisMarie Tremblay, Montréal, Presses de l'Université de Montréal, 1972, 288 pp.

Cet ouvrage comble incontestablement un vide en rassemblant pour la première fois et d'une façon systématique les prises de position et les orientations principales émanant des instances dirigeantes de la CSN et de la FTQ entre 1940 et 1970.

L'auteur étudie successivement l'idéologie de la CTCC-CSN (Confédération des travailleurs catholiques du Canada devenue en 1960 la Confédération des syndicats nationaux) et celle de la FPTQ-FUIQ-FTQ (La Fédération provinciale du travail du Québec et la Fédération des unions industrielles du Québec se sont fusionnées en 1957 pour devenir la Fédération des travailleurs du Québec). Dans les deux cas, la démarche utilisée est la même. Sous le titre «définition de soi 》 est analysée la conception que ces organisations syndicales se font de l'organisation syndicale et de l'action syndicale, de leurs interlocuteurs le patronat et l'Etat. Suivent chaque fois trois chapitres consacrés respectivement à «la pensée économique », à 《la pensée sociale», et aux 《 relations de travail ».

La documentation est abondante, ses sources indiquées avec une précision méticuleuse et c'est pourquoi ce livre est un instrument de travail indispensable pour quiconque se préoccupe du syndicalisme québécois et de son histoire.

Mais cette utilité marque simultanément les limites de cet effort de documentation.

Une première limite découle de ce que cette analyse «ne tient pas compte des structures intermédiaires et locales 》 (p. 19). L'auteur recommande lui-même qu'elle soit poursuivie à ces niveaux qui, dit-il, constituent aussi des centres de formation d'idéologie, « afin de déterminer le degré de concurrence idéologique entre les diverses structures ainsi que le degré d'interinfluence réciproque $\gg$ (pp. 19 et 20).

L'on ne peut que souhaiter que ce vœu soit exaucé, encore qu'il ne faudrait pas encourir le risque d'hypothéquer à l'avance le résultat de cette recherche en évoquant à priori quelque concordance entre les orientations idéologiques de ces diverses instances, ou encore en sous-estimant la difficulté qu'impliquerait l'interprétation du silence de certaines d'entre elles.

Dans la préface de ce livre Jean-Réal Cardin tranche cette dernière question en employant un ton nuancé. «En période de crise aiguë, d'affrontements irrémissibles, un hiatus entre la philosophie officielle et le sentiment de «la base », ou d'une partie de celle-ci, peut s'introduire momentanément: nous en avons des preuves lorsque des schismes surviennent ou que des réalignements profonds s'opèrent dans la composition des leaderships. Les événements récents dans le monde syndical québécois en sont un exemple. Mais ceci signifie, en même temps, qu'en règle très générale, et de surplus en période historique étendue, la dichotomie que d'aucuns voudraient toujours percevoir au sein des organismes syndicaux entre leur «pensée officielle » et celle de la 《majorité silencieuse » reste assez souvent plus imaginaire que réelle ».

Où sont les fondements objectifs d'une telle affirmation ? Faudrait-il la rapprocher de «ce sens historique du syndicalisme québécois 》 qu'évoque Louis-Marie Tremblay et par là de quelque vague philosophie de l'histoire inavouée mais dont seule l'histoire achevée pourrait confirmer le bien fondé ou plus simplement d'options qui n'ont rien à voir avec l'objectivité scientifique?

Ceci n'est cependant que broutille par comparaison avec la façon dont est traitée la question de l'idéologie et celle des conditions de son étude objective.

Certes il y a bien à la page 16 une allusion aux travaux de Fernand Dumont 
sur l'analyse des idéologies mais qui n'en respecte ni les nuances ni surtout les inquiétudes épistémologiques et la problématique, ce dernier abordant l'analyse des idéologies en les reférant le plus souvent à la culture dont elles constitueraient comme «le noyau agissant $\gg$.

Le schéma que nous propose LouisMarie Tremblay n'est en fait qu'un décalque mais caricatural des principes constitutifs de l'action et non pas des idéologies à la manière d'Alain Touraine. «Définition de soi-même, des autres et rapports entre soi et les autres $\gg$ renvoient aux principes d'identité, d'opposition et de totalité (cf. page 16).

Quelques paragraphes plus loin, LouisMarie Tremblay affirme : «C'est en établissant un rapport entre l'homme et les structures sociales, économiques et politiques que le syndicalisme évalue les structures sociales, économiques et politiques, définit ses objectifs et procède aux moyens d'action》 (p. 16), avant d'ajouter ce que sera son propre modèle d'analyse : «Le développement de l'idéologie est conditionné par les quatre facteurs suivants : les sources de tension, la nature du pouvoir, les facteurs de solidarité et les types de leadership (p. 16), et de suggérer que ces orientations - les orientations idéologiques sont l'une des dimensions constitutives de l'action syndicale.

Ainsi se trouvent allégrement tranchées des questions aussi controversées que décisives qui concernent aussi bien le statut épistémologique de l'idéologie soit ce rapport qu'elle entretient avec la vérité ou avec l'erreur, sa genèse soit l'ensemble des facteurs ou des conditions qui la produisent ou qui accompagnent sa naissance que sa fonction qui, dit-on, peut être de mystifier ou à l'inverse, de dévoiler des avenirs possibles.

Cette faiblesse interdit évidemment l'établissement de relations significatives et objectives entre idéologie et pratique syndicale, toute analyse du rôle des appareils syndicaux en tant que définisseurs d'idéologie, toute mesure de la cohérence de ces idéologies et de leur capacité de transformer les situations objectives.

Que reste-t-il, sinon un énoncé des thèmes, une classification qui n'est guère convaincante et l'esquisse d'une périodisation de l'évolution des idéologies syndicales qui laisse supposer une évolution que Jean-Réal Cardin systématise dans la préface comme une « radicalisation accrue $\gg$ sans que rien dans le texte permette de décider ou non de son caractère utopique ?

\section{Bernard SOLASSE}

British Trade Unions and the Problem of Change, par Will Paynter, Montréal, McGill-Queen's University Press, 1970, $172 \mathrm{pp}$.

L'auteur de cet ouvrage est ni un universitaire ni un habitué des cercles de dilettantes qui pontifient sur le syndicalisme et le monde du travail. Il a milité toute sa vie dans le syndicalisme. A la fin de la première guerre mondiale, il a commencé à travailler dans les puits d'une mine de charbon en Grande Bretagne et comme syndicaliste il a participé à toutes les luttes de son syndicat, aux grandes marches de la faim des mineurs de $1931,1932,1936$. Il a occupé la charge de président des South Wales Miners pendant un certain temps pour accéder ensuite au poste très important de secrétaire de la puissante National Union of Mineworkers. Les observations qu'il nous livre ici sur le syndicalisme sont le fruit de l'expérience d'un homme qui a vécu le syndicalisme anglais durant cinquante ans et qui lui a consacré le meilleur de ses énergies. Tout au long de son ouvrage il rappellera les événements auxquels il a été mêlé, les attitudes qui ont été prises et les résultats.

Pour ce vétéran, qui demeure encore socialiste convaincu, si le capitalisme reste encore le capitalisme, des changements sont intervenus qui nécessitent une adaptation des structures et des stratégies syndicales.

L'ouvrage est divisé en cinq parties : introduction; changements dans la structure économique ; structure des syndicats ; le pouvoir et la constitution du Trades Union Congress ; stratégie pour l'avenir.

Les principaux changements que l'auteur explique dans la structure économique sont la concentration industrielle, le professionnalisme dans la gestion, la révolution technologique, les syndicats d'employeurs, le rôle prépondérant de l'Etat dans l'industrie avec ses politiques 